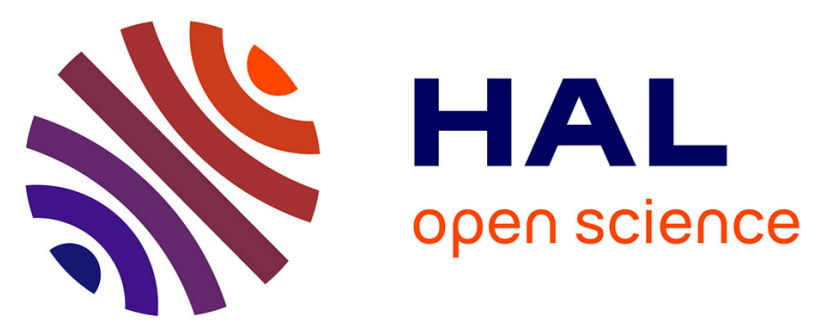

\title{
Transition metal silicide surface grafting by multiple functional groups and green optimization by mecanochemistry
}

Maxime Godfroy, Mahmoud Khalil, Claude Niebel, Thibaut Jarrosson, Dominique Foix, Valérie Flaud, Françoise Serein-Spirau, Romain Viennois, Michel Granier, Mickael Beaudhuin

\section{To cite this version:}

Maxime Godfroy, Mahmoud Khalil, Claude Niebel, Thibaut Jarrosson, Dominique Foix, et al.. Transition metal silicide surface grafting by multiple functional groups and green optimization by mecanochemistry. Physical Chemistry Chemical Physics, 2019, 21 (46), pp.25720-25727. 10.1039/C9CP03864G . hal-02997401

\section{HAL Id: hal-02997401 \\ https://hal.science/hal-02997401}

Submitted on 23 Nov 2020

HAL is a multi-disciplinary open access archive for the deposit and dissemination of scientific research documents, whether they are published or not. The documents may come from teaching and research institutions in France or abroad, or from public or private research centers.
L'archive ouverte pluridisciplinaire HAL, est destinée au dépôt et à la diffusion de documents scientifiques de niveau recherche, publiés ou non, émanant des établissements d'enseignement et de recherche français ou étrangers, des laboratoires publics ou privés. 


\title{
Transition metal silicide surface grafting by multiple functional
}

\section{groups and green optimization by mecanochemistry}

Maxime Godfroy ${ }^{\mathrm{a}}$, Mahmoud Khalil ${ }^{\mathrm{a}}$, Claude Niebel ${ }^{\mathrm{a}}$, Thibaut Jarrosson ${ }^{\mathrm{a}}$, Dominique Foix ${ }^{\mathrm{b}}$, Valérie Flaud $^{\text {a }}$, Françoise Serein-Spirau ${ }^{\mathrm{a}}$, Romain Viennois ${ }^{\mathrm{a}}$, Michel Granier ${ }^{\mathrm{a}, * 1}$, Mickaël Beaudhuin ${ }^{\mathrm{a}, *}$

${ }^{\text {a } I n s t i t u t ~ C h a r l e s ~ G e r h a r d t ~ M o n t p e l l i e r, ~ U M 5 ~ 5253, ~ U n i v . ~ M o n t p e l l i e r, ~ C N R S, ~ E N S C M, ~ M o n t p e l l i e r, ~}$ France

${ }^{\mathrm{b}}$ CNRS/Univ Pau \& Pays Adour / E2S UPPA, Institut des sciences analytiques et de physicochimie pour l'environnement et les matériaux, UMR 5254, 64000 Pau, France

\begin{abstract}
Chromium disilicide $\left(\mathrm{CrSi}_{2}\right)$ particles were synthesized by arc melting furnace followed by mechanical milling. XRD and DLS analyses show that aggregates of around $3 \mu \mathrm{m}$ containing about $10 \mathrm{~nm}$ sized crystallites were obtained. These aggregates were functionalized in solution by coupling agents with different anchoring groups (silane, phosphonic acid, alkene and thiol) in order to disperse them into an organic polymer. Dodecene was used to modify $\mathrm{CrSi}_{2}$ surface during mecano-synthesis in a grinding bowl with quite little solvent quantity and the optimization step allowed to reduce the aggregates size to $500 \mathrm{~nm}$. A thermoelectric composite was then made between alkene $\mathrm{CrSi}_{2}$ grafted samples and poly(pphénylène-2,6-benzobisoxazole). This study opens the route of new surface grafting of intermetallic silicides for applications linked to electronic and/or energy.
\end{abstract}

\footnotetext{
*Corresponding authors: mickael.beaudhuin@umontpellier.fr, michel.granier@umontpellier.fr
} 


\section{INTRODUCTION}

Nowadays, $55 \%$ of the electricity produced by heat engines and turbines is wasted into lost thermal energy ${ }^{1}$. Thermoelectric materials are a promising solution to convert this waste-heat into electricity. Indeed, they exhibit the advantage to have no moving parts as they are solidstate devices. Their efficiency is evaluated by the dimensionless thermoelectric figure of merit ZT which is defined as ZT $=\alpha^{2} \sigma \mathrm{T} / \lambda$ where $\alpha, \sigma, \mathrm{T}$ and $\lambda$ are the Seebeck coefficient, the electrical conductivity, the absolute temperature and the thermal conductivity respectively. The widely used devices are built from inorganic compounds that offer high electrical conductivity and high Seebeck coefficient. Nevertheless, good ZT of about 1 are only obtained by expensive, toxic and hard to process compounds such as $\mathrm{Bi}_{2} \mathrm{Te}_{3}(200-500 \mathrm{~K})$ and $\mathrm{PbTe}(500-800 \mathrm{~K})^{2,3}$. In order to improve the competitiveness of thermoelectric materials, it is necessary to develop new performant and cheap inorganic compounds. Silicides are really competitive in terms of cost that is less than $5 \$ / \mathrm{kg}$ compared to more than $100 \$ / \mathrm{kg}$ for $\mathrm{Bi}_{2} \mathrm{Te}_{3}{ }^{4}$. Among them, we have chosen to work with $\mathrm{CrSi}_{2}$ which presents the main advantage to have a power factor $\left(\mathrm{PF}=\alpha^{2} \sigma\right)$ similar to $\mathrm{Bi}_{2} \mathrm{Te}_{3}$ and $\mathrm{PbTe}$. Nevertheless, it possesses a relatively high thermal conductivity between 11 and $16 \mathrm{~W} \cdot \mathrm{m}^{-1} \cdot \mathrm{K}^{-1}$ at room temperature and between 7 and $12 \mathrm{~W} \cdot \mathrm{m}^{-1} \cdot \mathrm{K}^{-1}$ at $900 \mathrm{~K}$ conducting to a ZT of 0.025 at room temperature and more than 0.25 at $900 \mathrm{~K}^{5}$. Different strategies are possible to reduce the thermal conductivity of thermoelectric materials: alloying with heavy elements ${ }^{6-8}$, nanostructuring ${ }^{9-11}$ or combining nano-particles with organic polymers that are known to have low thermal conductivities below $1 \mathrm{~W} \cdot \mathrm{m}^{-1} \cdot \mathrm{K}^{-1}{ }^{12,13}$ can be noticed. In addition, using organic polymers is relevant because they are low-cost raw materials, easy to shape and their electrical properties can be easily modulated by changing their structure or the doping according to different ways by chemical or electrochemical processes ${ }^{14,15}$. Up to now, the most famous organic polymer is the PEDOT:PSS that becomes competitive when it is combined with inorganic materials exhibiting electrical conductivity that can achieve $5400{\mathrm{~S} . \mathrm{cm}^{-1}}^{14}$ and a ZT as high as $0.42^{12}$. A recently developed strategy to increase the figure of merit ZT is to take the advantages of inorganic materials (high Seebeck coefficient and electrical conductivities) and 
organic polymers (low thermal conductivities). Some examples of inorganic materials/organic polymers composites have recently been tested ${ }^{16,17}$ but only a part of them show a synergistic effect of the composite compared to the separated elements such as PEDOT:PSS passivated Te nanorods ${ }^{18}$. However, some papers pointed out its low moisture and thermal stability associated to the PSS doping ${ }^{19,20}$. To answer to this problem, we decided to choose the most thermally stable organic polymer known in the literature under nitrogen atmosphere $(>900 \mathrm{~K})$, the poly $(\mathrm{p}-$ phenylene-benzobisoxazole) (PBO) ${ }^{21}$ to develop our composite films. We will see later in this paper that the composite films have poor mechanical properties even at low concentrations of $\mathrm{CrSi}_{2}$. It was then necessary to investigate the functionalization of silicides $\mathrm{CrSi}_{2}$ particles before their dispersion in the organic matrix and this was done with different coupling agents. We believe that the inorganic nanomaterials functionalization can enhance their interaction with organic polymers by Van der Waals interactions and this could permit to explore high inorganic/organic ratios. Furthermore, it is possible to graft coupling agents with terminal hanging groups that could react for example by click-chemistry with functional moieties carried by the organic polymers ${ }^{22}$.

Many functional groups could be used to functionalize $\mathrm{CrSi}_{2}$ surfaces due to the presence of two different atoms. Phosphonic acids are largely used in the functionalization of metal oxide surfaces such as $\mathrm{TiO}_{2}, \mathrm{ZrO}_{2}{ }^{23}, \mathrm{ZnO}^{24}$, ITO (Indium Tin Oxide) ${ }^{25}, \mathrm{SnO}_{2}{ }^{26}$ and one example is reported for phosphonic acid group grafting on Co-Cr alloy oxide layer ${ }^{27}$. The interest of silane groups is well established as they are used in many applications such as chromatography, liquid crystal displays and microelectronics. The silane groups can functionalize a wide variety of substrates from carbon black to silica. On inorganic oxides $\left(\mathrm{Fe}_{2} \mathrm{O}_{3}, \mathrm{TiO}_{2}, \mathrm{Cr}_{2} \mathrm{O}_{3}\right)$ and on silicon ${ }^{22,28}$ the effectiveness of the grafting ranges from poor to excellent effectiveness ${ }^{22,28}$. The thiol group is more acknowledged to be efficiently grafted on gold nanoobjects ${ }^{29,30}$ and also on silver ${ }^{31,32}$; it was recently attempted to be grafted on silicon nanocrystals but a poor stability towards water or oxygen was recorded ${ }^{33}$. Finally, alkene moieties were largely used to functionalize silicon surfaces to prevent from the formation of a native oxide surface especially in the domain of electronics 34,35 . Thus, long dodecane hydrophobic chains bearing a phosphonic acid, 
triethoxysilane, thiol or alkene functionalities were chosen to modify $\mathrm{CrSi}_{2}$ particles surface with the aim to make them more compatible with soluble organic materials soluble in organic solvent. Interestingly, the four-studied different functional groups have been successfully grafted on the inorganic particles. These newly grafted objects have been characterized by XPS and FTIR and hydrophobicity tests were realized to show the grafting efficiency. In the following, the work will be focused on the alkene moiety coupling agents and on its possible direct grafting in a grinding bowl. This enables to reduce the experiments time and to take benefit from the heat delivered by the milling balls to favour the coating. Finally, we show that grafting with coupling agent improves significantly the mechanical properties of $\mathrm{PBO} / \mathrm{CrSi}_{2}$ composites.

\section{RESULTS AND DISCUSSION}

\section{Synthesis of $\mathrm{CrSi}_{2}$ particles}

The obtained particles were characterized by XRD that showed a pure hexagonal $\mathrm{CrSi}_{2}$ phase (Figure 1-left). The Rietveld refinements were performed on the XRD patterns in the $2 \theta$-range of $20-110^{\circ}$.

The estimated grains size ranges between 10-16 nm according to the Williamson-Hall formula for the mechanical milled nanocrystallites. These sizes were in agreement with our previous works $^{7,9}$. Moreover, the mechanical milled nanocrystallites have been characterized by BET that informed us about the rather low specific surface of $2.67 \mathrm{~m}^{2} / \mathrm{g}$ that could be explained by the presence of large aggregates as observed by SEM (Figure 1-right) with sizes between 200 $\mathrm{nm}$ and more than 2 microns. These aggregates were confirmed by DLS with an average size of around 3 microns. TEM images showed us that the aggregates were mainly composed of nanocrystallites with a size comprised between 6 and $10 \mathrm{~nm}$, in adequacy with XRD analysis (see S.I. Fig. S1).

To improve further interactions between inorganic particles and organic materials different coupling agents were investigated. 

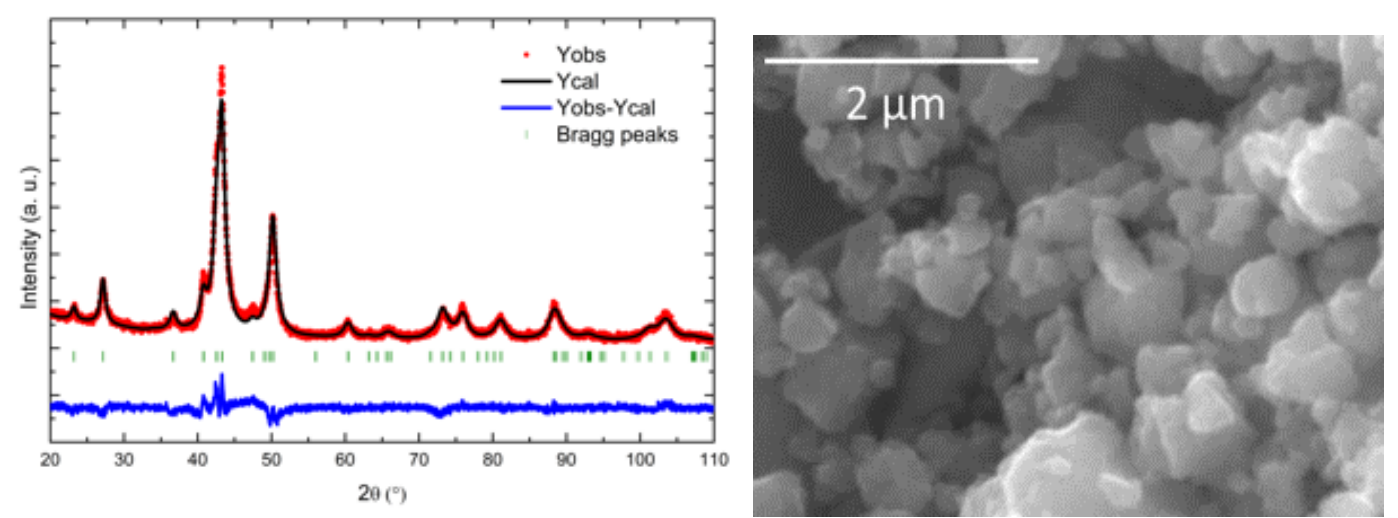

Fig. 1 (Left) Powder X-Ray diffraction of $\mathrm{CrSi}_{2}$ alloys obtained by mechanical alloying and refined with Fullprof software. The experimental $\left(\mathrm{Y}_{\mathrm{obs}}\right)$, calculated $\left(\mathrm{Y}_{\text {calc }}\right)$ and residual fit $\left(\mathrm{Y}_{\mathrm{obs}}{ }^{-}\right.$ $\mathrm{Y}_{\text {calc }}$ ) is given as well as the Bragg peaks. (Right) SEM image of mechanical milled $\mathrm{CrSi}_{2}$ powders

\section{Functionalization of particles with coupling agents and their characterization}

As we introduced, the $\mathrm{CrSi}_{2}$ particles functionalization was attempted with long hydrophobic dodecane chains with a phosphonic acid, triethoxysilane, thiol or alkene anchoring group. Indeed, each coupling agent was expected or not to be grafted either on chromium or on silicon with a passivated oxide surface (Scheme 1).
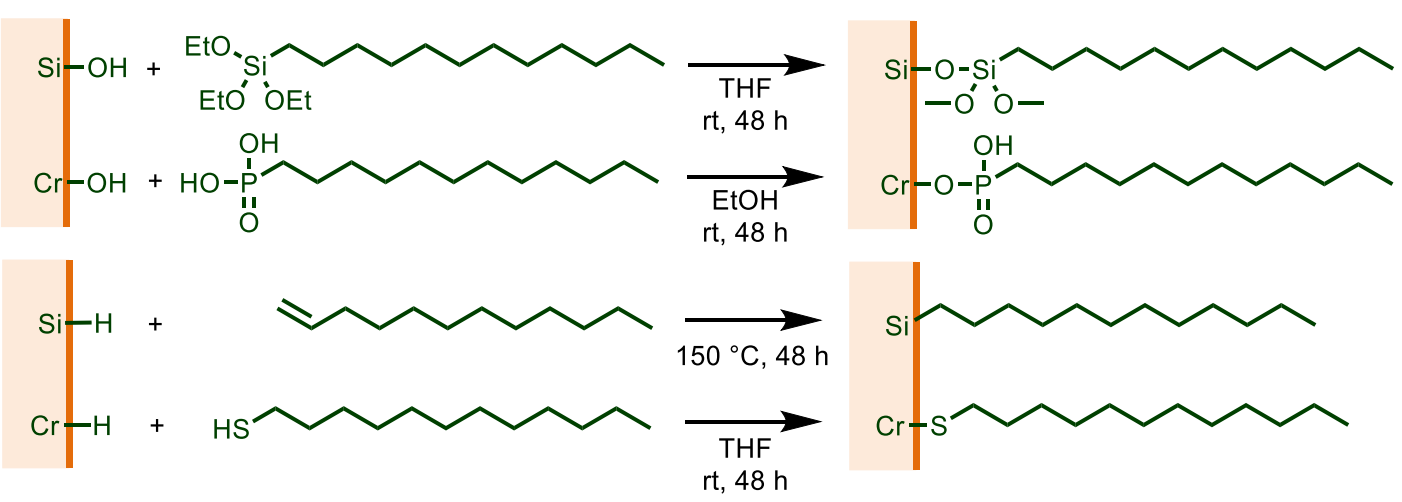

Scheme 1 Functionalization of $\mathrm{CrSi}_{2}$ by different coupling agents: dodecyltriethoxysilane, dodecylphosphonic acid, 1-dodecene and 1-dodecanethiol. 
Before XPS and FTIR characterization on the coated particles, a hydrophobic test was carried out. It consisted in introducing a small quantity of nanocrystallites in a hemolysis tube subsequently filled with distilled water. The results are given in Figure 2 and it is observed that no-grafted $\mathrm{CrSi}_{2}$ particles sink whereas grafted $\mathrm{CrSi}_{2}$ particles float on water. This is due to the oxide surface that gives a hydrophilic character to these particles.

In all attempts of grafting, $\mathrm{CrSi}_{2}$ particles stayed at the surface and no crystallites sank. This can be explained by the hydrophobicity of dodecyl chains that enable particles to float on water.
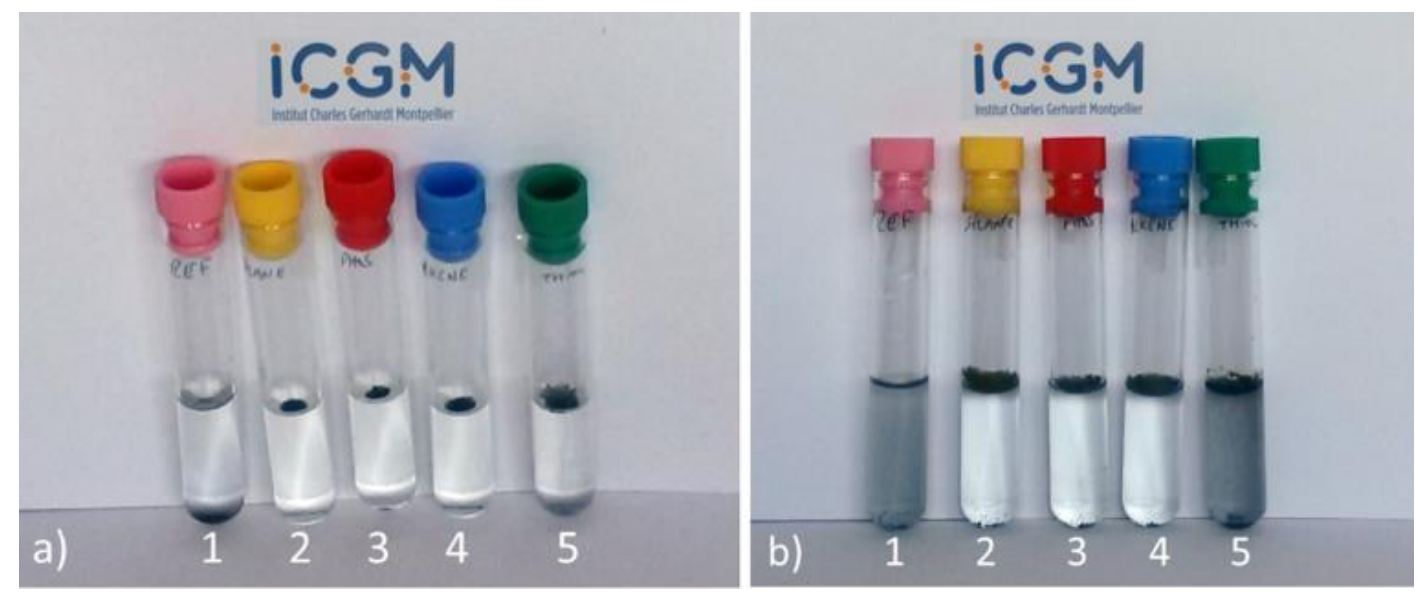

Fig. 2 Tests of $\mathrm{CrSi}_{2}$ particles in water, a) Before stirring. b) After stirring. 1) No-grafted nanocrystallites. Nanocrystallites functionalized with 2) dodecyltriethoxysilane, 3) ndodecylphosphonic acid, 4) 1-dodecene and 5) 1-dodecanethiol

To check the stability of the grafting, hemolysis tubes stirring was vertically done for ten seconds. The only particles which sank into the water were the ones grafted with dodecanethiol ones, (Figure 2b, 3). Similar behavior has been observed by Yu et al. ${ }^{33}$ after only 20 seconds in distilled water, silicon nanocrystals grafted with dodecanethiol lost emissive properties that the authors explained as the result of the surface oxidation. Thus, the water induces the loss of the thiol-functionalized chains from the silicon surface. One also notices that the functionalized chains of alkene, phosphonic acid and silane are stable for a long period either in water or $\mathrm{MeOH} /$ water mixtures (see below). Indeed, after more than 12 months in-hemolysis tubes, the $\mathrm{CrSi}_{2}$ particles are still floating. 
In addition to this simple hydrophobic test, the functionalized nanocrystallites were introduced in hemolysis tubes filled with different ratios of $\mathrm{MeOH} /$ water which is generally referred to the methanol number test (Figure 3).

The more the particles stay at the surface for higher $\mathrm{MeOH} /$ water ratios, the more the particles are highly coated by hydrophobic coupling agents.
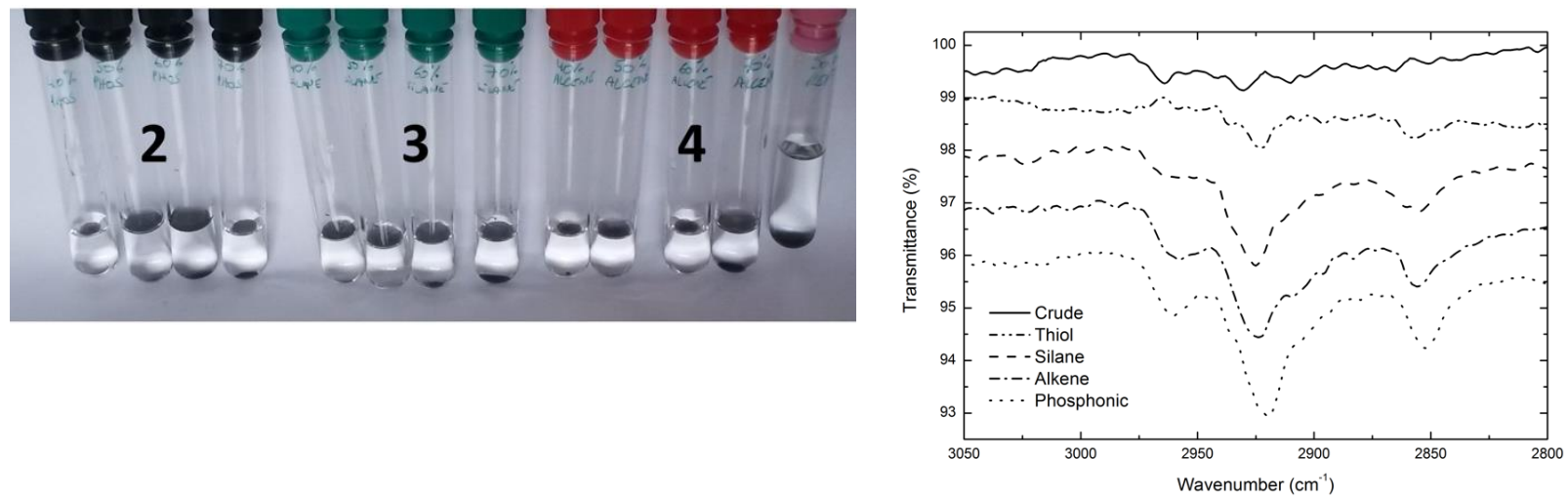

Fig. 3 Tests of $\mathrm{CrSi}_{2}$ particles in methanol/water mixtures. The different functionalized particles with 2) phosphonic acid, 3) silane and 4) alkene coupling agent were introduced in $\mathrm{MeOH} /$ water mixtures with $40 \%, 50 \%, 60 \%$ and $70 \%$ of methanol.

\begin{tabular}{|c|c|c|c|c|}
\hline Grafting agent & No grafted & Alkene & $\begin{array}{c}\text { Phosphonic } \\
\text { acid }\end{array}$ & Silane \\
\hline $\mathrm{C}_{1 \mathrm{~s}}$ & 33.26 & 42.73 & 37.34 & 44.53 \\
\hline $\mathrm{Cr}_{2 \mathrm{p}}$ & 5.02 & 3.34 & 3.22 & 2.66 \\
\hline $\mathrm{Si}_{2 \mathrm{p}}$ & 25.01 & 29.34 & 26.56 & 21.61 \\
\hline $\mathrm{O}_{1 \mathrm{~s}}$ & 36.71 & 24.58 & 31.77 & 31.20 \\
\hline $\mathrm{P}_{2 \mathrm{p}}$ & & & 1.11 & \\
\hline
\end{tabular}

Table 1. Atomic percentage of the elements present in $\mathrm{CrSi}_{2}$ powders calculated from XPS spectra, no grafted or grafted with either alkene, phosphonic acid or silane. 
The methanol number is defined by the percentage of methanol in methanol/water mixture where no particles sank. Thus, the methanol number of the particles functionalized with phosphonic acid is $\sim 50 \%, \sim 50 \%$ with silane and $\sim 60 \%$ with alkene. This indicated that the alkene-coated $\mathrm{CrSi}_{2}$ particles are the most densely packed functionalized nanocrystallites.

FTIR characterizations are given in figure 4, no signal of any C-H bonds was observed in the 2800-3000 $\mathrm{cm}^{-1}$ region for the ungrafted $\mathrm{CrSi}_{2}$ while $\mathrm{C}_{\mathrm{sp} 3} \mathrm{CH}_{2}$ symmetrical stretching vibration around $2855 \mathrm{~cm}^{-1}$ and $\mathrm{C}_{\mathrm{sp} 3} \mathrm{CH}_{2}$ asymmetrical stretching vibration between 2920 and $2925 \mathrm{~cm}^{-1}$ were well revealed on the grafted $\mathrm{CrSi}_{2}$ nanocrystallites. Furthermore, the $\mathrm{C}_{\mathrm{sp} 3} \mathrm{CH}_{3}$ unsymmetrical stretching vibration around $2960 \mathrm{~cm}^{-1}$ could be seen for silane, alkene and phosphonic acid-grafted $\mathrm{CrSi}_{2}$ nanoobjects. As these measurements were done several weeks after the grafting of $\mathrm{CrSi}_{2}$ particles, the low signal observed for thiol-functionalized $\mathrm{CrSi}_{2}$ can be explain by its instability under air.

In table 1 , the atomic percentage of the elements presents in $\mathrm{CrSi}_{2}$ powders are calculated from XPS spectra and showed the presence of high $\mathrm{C}$ and $\mathrm{O}$ amount on no-grafted sample, whereas $\mathrm{C}$ come from external contamination, $\mathrm{O}$ found at the surface of the powder signifies that the surface is oxidized. It is known that $\mathrm{CrSi}_{2}$ is stable in air up to $973 \mathrm{~K}^{36,37}$ due to the formation of a protective layer either of $\mathrm{Cr}_{2} \mathrm{O}_{3}$ or $\mathrm{SiO}_{2}$ which limits the oxygen diffusion. The peaks at $103 \mathrm{eV}$ and, 577 and $587 \mathrm{eV}$ attributed to oxidized silicon and oxidized chromium respectively confirm the presence of this oxide layer. 


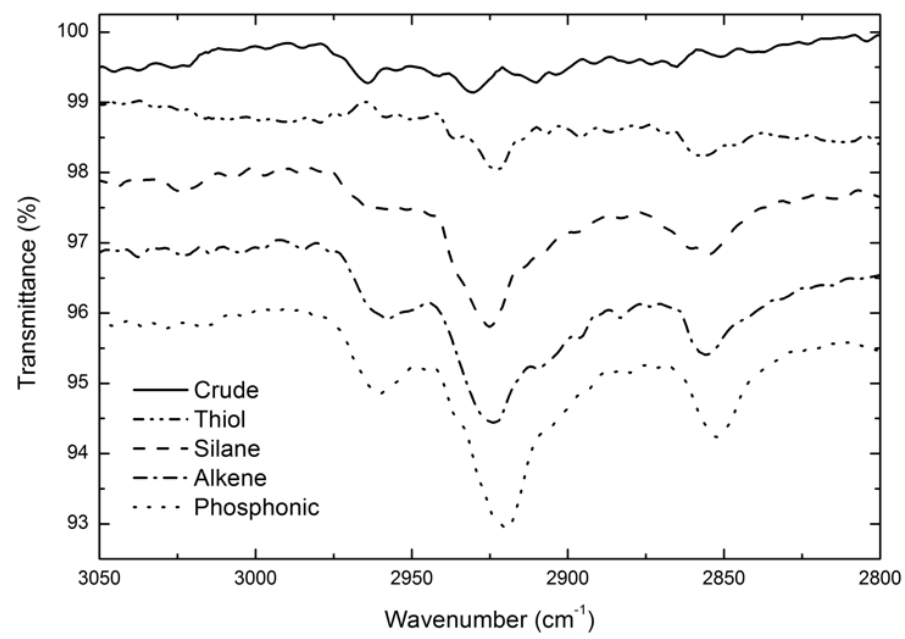

Fig. 4 FTIR spectra of ungrafted (crude) compared to grafted $\mathrm{CrSi}_{2}$ nanocrystallites.

It is then interesting to notice that the oxygen concentration decreases after grafting meaning that the grafting agent protects, at least partially, the surface from oxidation. These results also showed clearly the presence of phosphorus bonding $\mathrm{P}_{2 \mathrm{p}}$ at $134.1 \mathrm{eV}$ (see S.I. Fig. S2) in the attempt of functionalization of $\mathrm{CrSi}_{2}$ particles with $\mathrm{n}$-dodecylphosphonic acid. The ratio of carbon in grafted nanoparticules with dodecene (43\%), with dodecylphosphonic acid (37\%) and with dodecyltriethoxysilane (45\%), were all higher than those of the no-grafted reference (33\%), which confirmed the long carbon chains coatings. 
To highlight the efficiency of the grafting process, it has then been also decided to graft a molecule containing a heteroatom clearly visible via XPS such as the fluorine. This heteroatom has a very clear XPS signature and has the advantage to be missing from the raw material surface. The chosen grafted molecules are the same as the ones described in scheme 1 , but all the $-\mathrm{CH}_{2} /-$ $\mathrm{CH}_{3}$ groups are replaced by $-\mathrm{CF}_{2} /-\mathrm{CF}_{3}$ ones. The XPS analyses show that this fluorine element is present on the four treated surfaces (S.I. Fig. S3 and S4). For the thiol-grafting, the amount of detected fluorine is very low. These surface analyses allow to conclude that the grafting process is efficient for the three fluorine molecules, in total agreement with the water immersion tests.

On both families of coupling agents (carbon or fluorine chain), XPS measurements did not highlight the presence of the sulfur on thiol-functionalized $\mathrm{CrSi}_{2}$ nanocrystallites, which confirms the coupling agent removing from the surface under ambient atmosphere containing water. All the investigations performed on narrow-range $C_{1 s}$ spectra did not permit to determine the type of bondings between $\mathrm{CrSi}_{2}$ and the grafting agent due to the high amount of $\mathrm{C}-\mathrm{C}$ bondings.

\section{Optimization of the alkene functionality grafting coupling agent}

For thermoelectric properties, it is necessary to have a material as conductive as possible, oxide surface must therefore be avoided due to their inherent insulating properties.

As phosphonic acid and silane groups are known to react with oxidized surfaces and thiol group did not permit to obtain stable coatings, the best coupling agent to graft $\mathrm{CrSi}_{2}$ appears to be alkene moiety since it reacts with non-oxidized hydrogen-terminated silicon surface. Nevertheless, the induced hydrosilylation reaction mostly takes place at $150-200{ }^{\circ} \mathrm{C}$ in the presence of pure alkene or alkene high concentrated solutions for several hours. Hallmann et al. have described the high-energy ball milling as a simple method to functionalize silicon nanoparticles with different alkene and alkyne groups ${ }^{38}$.

We first studied, for reference, the effect of milling time and of the presence of solvent on the average crystallites size and on the average aggregates size in Table 2, entries 1 to 4 . Then we attempted to graft our $\mathrm{CrSi}_{2}$ nanocrystallites directly in the grinding ball in Table 2 entries 5 to 
8 and, as expected, all of our carried-out experiments showed an efficient recovering. From these experiments we found that a decrease of the aggregates size also leads to a strong decrease of the polydispersity.

In entries 1 and 5, the arc melted button is milled for $4 \mathrm{~h}$ respectively with or without grafting agent. The average aggregate size strongly decreases from 3000 to $400 \mathrm{~nm}$ whereas the nanocrystallite size increases from 10 to $80 \mathrm{~nm}$. This result can be explained by the possible lubrication property of the dodecyl chain.

So, although this method permits to strongly decrease the average aggregates size, in order to maintain a small average crystallite size, it is important to firstly mill the arc melted button in order to obtain crystallite size already about $10 \mathrm{~nm}$. Then, pure dodecene can be added in the vessel, with or without solvent, and sealed under argon (all the process was performed in a glovebox), followed by $4 \mathrm{~h}$ milling (entry 6 to 8 ).

This is what has been performed in entries 2 and 6, we found that the crystallite size is similar whereas the aggregate size decreases from about 2800 to $500 \mathrm{~nm}$. This result indicates here again that the alkene solution has a strong impact on the average aggregates size.

Then, as we observed in entries 3 and 4 that the use of solvent lead to a strong decrease of the average aggregates size, we attempted to reduce again the aggregates size by using a solvent with dodecene (entries 7 and 8), and to look for a cumulative effect. We found that the average crystallite size is not modified nor the average aggregates size, however, our XRD pattern are much less intense as if the coverage was denser and that the quantity of alkyl chains hides the signal of $\mathrm{CrSi}_{2}$. This was also observed by Hessel et al. in the case of alkene-functionalized silicon nanoparticles ${ }^{39}$. In conclusion, the functionalization is important to break the aggregates of nanocrystallites but this could be only obtained on previously milled buttons. 


\begin{tabular}{|c|c|c|c|c|c|}
\hline Entry & $\begin{array}{c}\text { Extra } \\
\text { milling }\end{array}$ & dodecene & heptane & $\begin{array}{c}\text { Average crystallites } \\
\text { size }^{\mathbf{a}}(\mathbf{n m})\end{array}$ & $\begin{array}{c}\text { Average aggregates } \\
\text { size / PDI }\end{array}$ \\
\hline $\mathbf{1}$ & - & - & - & 10 & $3000 / ? ?$ \\
\hline $\mathbf{2}$ & $4 \mathrm{~h}$ & - & - & 13 & $2800 / 1.50$ \\
\hline $\mathbf{3}$ & $4 \mathrm{~h}$ & - & $1.6 \mathrm{~mL}$ & 11 & $1600 / 1.24$ \\
\hline $\mathbf{4}$ & $4 \mathrm{~h}$ & - & $4.8 \mathrm{~mL}$ & 10 & $400 / 0.65$ \\
\hline $\mathbf{5}$ & - & $800 \mu L$ & - & 80 & $500 / 0.72$ \\
\hline $\mathbf{6}$ & $4 \mathrm{~h}$ & $800 \mu L$ & - & 9 & $500 / 0.75$ \\
\hline $\mathbf{7}$ & $4 \mathrm{~h}$ & $160 \mu L$ & $1.6 \mathrm{~mL}$ & 9 & $900 / ? ?$ \\
\hline $\mathbf{8}$ & $4 \mathrm{~h}$ & $160 \mu L$ & $4.8 \mathrm{~mL}$ & 10 & \\
\hline
\end{tabular}

Table 2 Effect of the solvent, coupling agent and extra milling on standard sample obtained after 4h button mechanical millings. (a) Cristallite size determined by Rietveld refinement on XRD data. b) Average aggregates size and its polydispersity, also note PDI, determined by DLS in EtOH.)

\section{Thermoelectric composite}

In figure 5, the inorganic-organic composites are presented for two different volume ratio $\mathrm{CrSi}_{2} / \mathrm{PBO}(50 / 50$ and $75 / 25)$ after heat treatment at $400^{\circ} \mathrm{C}$ for $1 \mathrm{~h}$. One notice that not grafted $\mathrm{CrSi}_{2}$ dispersed in the PBO lead to films with numerous cracks and by an exfoliation of the composite from the glass substrate. At the contrary, $\mathrm{CrSi}_{2}$ grafted particles dispersed in the $\mathrm{PBO}$ polymer gives relatively uniform films with no cracks and good adhesion with the substrate. 


\begin{tabular}{|c|c|c|}
\hline $\begin{array}{c}\mathrm{CrSi}_{2} / \text { PBO } \\
\text { Volume } \\
\text { Ratio }\end{array}$ & Not Grafted & Grafted \\
\hline $50 / 50$ & \\
\hline $75 / 25$ & & \\
\hline
\end{tabular}

Fig. 5 Not grafted $\mathrm{CrSi}_{2}$ particles and alkene grafted $\mathrm{CrSi}_{2}$ particles for two volume ratio of $\mathrm{CrSi}_{2} / \mathrm{PBO}(50 / 50$ and $75 / 25)$

In figure 6-a, it is observed that the not grafted $\mathrm{CrSi}_{2}$ particles composite film is heterogeneous with two different regions, the bottom part of the composite is composed with $\mathrm{CrSi}_{2}$ particles and a low amount of PBO whereas the upper part is mainly composed of PBO and a low amount of $\mathrm{CrSi}_{2}$ particles. At contrary, in figure 6-b the composite film is homogeneous and the alkene grafted $\mathrm{CrSi}_{2}$ particles are well dispersed with the PBO. We believe that it can be explained by the presence of long alkyl chains on $\mathrm{CrSi}_{2}$ particles which bring a better interaction with the organic polymer, increasing its dispersion. The electrical resistivity of the grafted films at room temperature is about $1 \mathrm{k} \Omega . \mathrm{cm}$ which is 1000 times higher than pure $\mathrm{CrSi}_{2}$ however, the Seebeck coefficient is similar to that of pure $\mathrm{CrSi}_{2}$ with $80 \mu \mathrm{V} / \mathrm{K}$. This led to a relatively low power factor compared to bulk $\mathrm{CrSi}_{2}$. Consequently, although the thermal conductivity is expected to be quite close to the polymer thermal conductivity, the figure of merit is somewhat negligible. These results were expected as the alkyl chains are not electrically conductive and because the HOMOLUMO of the polymer has not been adjusted with the inorganic compound. The expected percolation of $\mathrm{CrSi}_{2}$ particles, even at large $\mathrm{CrSi}_{2}$ amount, was consequently not reached. 

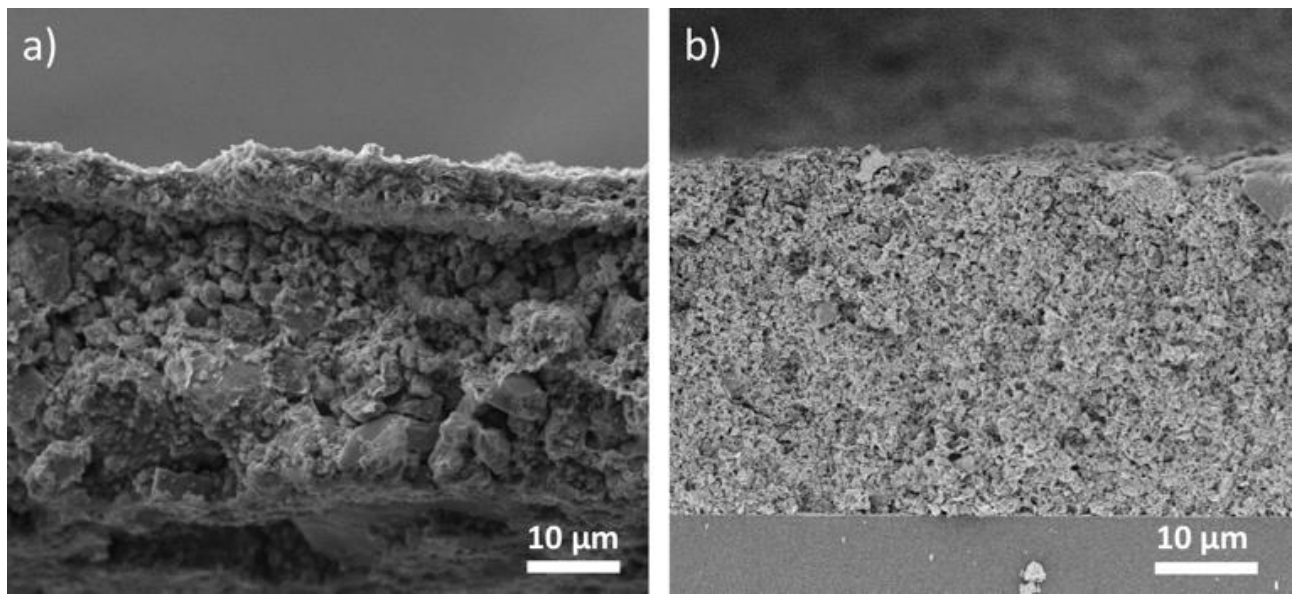

Fig. 6 SEM images obtained with secondary electron of $75 / 25 \mathrm{CrSi}_{2} / \mathrm{PBO}$ volume ratio for a) not grafted $\mathrm{CrSi}_{2}$ particles and b) alkene grafted $\mathrm{CrSi}_{2}$ particles

\section{CONCLUSIONS}

We have seen that it is possible to obtain pure $\mathrm{CrSi}_{2}$ nanocristallites by combining arc-melting and mechanical alloying. The as-obtained powder is made of aggregates $(\sim 3 \mu \mathrm{m})$ of nanocristallites $(\sim 10 \mathrm{~nm})$. Two methods to functionalize the chromium disilicide powders were reported, in solution or in a grinding bowl. In solution, the successful functionalization of $\mathrm{CrSi}_{2}$ particles has been described with 3 different coupling agents: alkene, silane and phosphonic acid grafting was confirmed by XPS and FTIR. The stability of the covalent interactions involved were also evaluated. A second optimized procedure involving the direct grafting of an alkene coupling agent in the grinding bowl enabled to save time and energy compared to the more conventional solution processing. Furthermore, this mechanochemical procedure enables to reduce the size of the aggregates from $3 \mu \mathrm{m}$ down to $500 \mathrm{~nm}$ together with being greener due to the strong decrease of the amount of solvent used. These new coated $\mathrm{CrSi}_{2}$ particles have been used in a mixture with organic polymers to realize inorganic-organic composites for thermoelectric applications. The obtained composites have a better mechanical behavior with grafted $\mathrm{CrSi}_{2}$ which open the routes to new high temperature thermoelectric applications using thermoelectric composites. Their limited electronic properties coming from the long alkyl chain 
could be compensated by working with pi-conjugated chain and by adjusting the HOMO-LUMO of the polymer with the inorganic alloys. These results could be easily extended to other transition metal silicides for applications not only limited to thermoelectric applications but also to electronic, photovoltaic.

\section{EXPERIMENTAL SECTION}

\section{Materials}

Chromium pieces (3-8 mm, 99,99\%) and silicon lump (99,9999\%) were purchased from Alfa Aesar. 1-Dodecene (95\%) was purchased from Janssen Chimica. n-Heptane (99\%) was purchased from Sigma Aldrich. N-N-dimethylallylamine (98\%) was purchased from Acros Organics. n-dodecylphosphonic acid from Sikemia. Dodecyltriethoxysilane and 1-dodecanethiol from Sigma Aldrich. All reactants and solvents were used as received.

\section{Synthesis of $\mathrm{CrSi}_{2}$ particles}

In order to avoid external contamination either during the sample preparation or the milling, all the process described below is performed under argon atmosphere. $\mathrm{CrSi}_{2}$ alloys were prepared by arc-melting technique under argon atmosphere using high purity $\mathrm{Cr}$ pieces (99.99\%) and very high purity Si lumps $(99.9999 \%)$ in a button shape $(\approx 2.7 \mathrm{~g}$ each). Sample homogeneity was improved by melting the samples four times with a turnaround between each melting. The buttons were crushed and grinded in an agate mortar pestle before being introduced into a $45 \mathrm{~mL}$ $\mathrm{Si}_{3} \mathrm{~N}_{4}$ grinding bowl with $5 \mathrm{Si}_{3} \mathrm{~N}_{4}$ balls $(15 \mathrm{~mm}$ diameter). Vessels were sealed under argon atmosphere in a glovebox. The milling was carried out with a "Fritsh Pulverisette 7 classic line", planetary micromill. The speed of the supporting disc and of the grinding bowl was, respectively, fixed to $650 \mathrm{rpm}$ and $1300 \mathrm{rpm}$ for all the experiments corresponding to a ball acceleration of about $147 \mathrm{~m} . \mathrm{s}^{-2}$. The ball to powder ratio was fixed to 10:1 for all the experiments.

\section{Functionalization of $\mathrm{CrSi}_{2}$ particles in Schlenk tubes}


Schlenk tubes were filled in a glovebox under argon atmosphere with $400 \mathrm{mg}$ of freshly synthesized $\mathrm{CrSi}_{2}$ particles. Schlenk tubes were sealed and were taken off from the glovebox.

- Procedure for n-dodecylphosphonic acid, dodecyltriethoxysilane and 1-dodecanethiol grafting: $10 \mathrm{~mL}$ of a $10^{-2} \mathrm{M}$ solution of the coupling agent in THF or EtOH (for $\mathrm{n}$ dodecylphosphonic acid) were transferred into the Schlenk tube and the solution was stirred 4 days at room temperature. The suspension was centrifuged at $20000 \mathrm{rpm}$ for 15 minutes and the supernatant was removed. Modified $\mathrm{CrSi}_{2}$ particles were washed three times with $\mathrm{CHCl}_{3}$ under sonication for 5 minutes. Particles were separated from the washing solvent by centrifugation at $20000 \mathrm{rpm}$ for 15 minutes. The resulting powders were dried under vacuum for one hour.

- Procedure for 1-dodecene grafting: $5 \mathrm{~mL}$ of 1-dodecene was added in the Schlenk tube and the solution was stirred 4 days at $150{ }^{\circ} \mathrm{C}$. After cooling at room temperature, the suspension was centrifuged at $20000 \mathrm{rpm}$ for 15 minutes and the supernatant was removed. Modified $\mathrm{CrSi}_{2}$ particles were washed three times with $\mathrm{CHCl}_{3}$ under sonication for 5 minutes. Particles were separated from the washing solvent by centrifugation at $20000 \mathrm{rpm}$ for 15 minutes. The resulting powders were dried under vacuum for one hour.

The procedure is similar for coupling agent with fluorine chain.

\section{Functionalization of $\mathrm{CrSi}_{2}$ particles by mechano-chemistry with 1-dodecene}

Freshly synthesized $\mathrm{CrSi}_{2}$ particles $(2.7 \mathrm{~g})$ were transferred into a glovebox. 1-Dodecene (700 $\mu$ mol) was introduced in the $\mathrm{Si}_{3} \mathrm{~N}_{4}$ vessel in presence or in absence of heptane (different volumes). The vessel was sealed in the glovebox and was introduced in the planetary mill for different times mentioned in the main text in Table 2. The treatment of the suspension of particles was identical to the one previously mentioned for the functionalization in solution.

\section{Synthesis of poly(p-phénylène-2,6-benzobisoxazole)}


The highly thermal resistive poly(p-phenylene-2,6-benzobisoxazole) (PBO) films were prepared following the optimized procedure described by Fukumaru et al. ${ }^{21}$. A TBS-prePBO precursor, soluble in most organic solvents, was obtained in two steps from commercially available 4,6diaminoresorcinol. Films of this soluble PPBO precursor could then be easily spin-coated on glass substrates, and a subsequent thermal treatment at $400^{\circ} \mathrm{C}$ for $1 \mathrm{~h}$ provided the PPBO films, which were characterized by ${ }^{1} \mathrm{H}$ NMR, IR spectroscopy and by thermogravimetric analysis (see SI Figs. S4 to S7).

\section{Thermoelectric composite}

The soluble precursor TBS-prePBO was dissolved at a concentration of $50 \mathrm{mg} \cdot \mathrm{mL}^{-1}$ in N,Ndimethylacetamide. The $\mathrm{CrSi}_{2}$ nanoparticles were added to give the expected ratio in percentage by volume and the mixture was sonicated for $30 \mathrm{~min}$ being used. Glass substrates of $12.5 \mathrm{x} 12.5$ $\mathrm{mm}$ were successively sonicated in acetone and in isopropanol for 10 minutes in each case, before being dried with dry air. The mixture of $\mathrm{CrSi}_{2} / \mathrm{TBS}$-prePBO was drop-casted $(50 \mu \mathrm{L})$ on glass substrates and the substrates were progressively heated at $400{ }^{\circ} \mathrm{C}$ with a ramp of $10{ }^{\circ} \mathrm{C}$ per minute then were let at $400{ }^{\circ} \mathrm{C}$ for one hour in a furnace under air. The resulting film were characterized electrically by Van der Paw technic with an homemade apparatus after deposition of gold plot. The Seebeck coefficient has been determined with an homemade apparatus.

\section{Powder X-ray Diffraction}

The powders were analyzed by using an X-Ray diffraction apparatus (Philips X'PERT, Cu-K radiation $1.5406 \AA$ with an accelerated detector PW3050/60 at $45 \mathrm{kV}$ and $30 \mathrm{~mA}$ settings). Rietveld refinement with Fullprof software ${ }^{40}$ was used for structural analysis of each sample.

\section{Brunauer-Emmett-Teller (BET)}


$\mathrm{N}_{2}$ sorption analyses were performed at $77 \mathrm{~K}$ using a Micromeritics Tristar 3000, equipped with automated surface area and pore size analyzer. Before analysis, samples were degassed at $250^{\circ} \mathrm{C}$ for $7 \mathrm{~h}$ using a Micromeritics VacPrep 061 degasser. BET surface areas were determined in the relative pressure range $\mathrm{P} / \mathrm{P}_{0}$ from 0.01 to 0.03 .

\section{Dynamic Light Scattering (DLS)}

DLS measurements were performed on a Vasco Particle Size Analyzer DL135 from Cordouan Technologies and analyzed with nanoQ software.

Fourier Transform InfraRed (FT-IR) Spectroscopy. ATR infrared spectra were recorded on a FTIR Perkin-Elmer 2000 spectrometer equipped with narrow band liquid nitrogen cooled MCT detector and a multireflexion ATR accessory. The sample compartment was purged with dry air. All the spectra were recorded at a resolution of $4 \mathrm{~cm}^{-1}$, and 128 scans were accumulated. The size of the silicon ATR crystal was 70x10x1 and 35 reflections were used.

\section{X-ray Photoelectron Spectroscopy}

XPS analysis was performed using a ESCALAB 250 Thermo Electron apparatus with Al K $\alpha$ $(1486.6 \mathrm{eV})$ as an excitation source and with a Kratos Axis Ultra spectrometer, using focused monochromatic Al $\mathrm{K} \alpha$ radiation $(\mathrm{h} v=1486.6 \mathrm{eV})$. For the ESCALAB 250 Thermo Electron apparatus, a surface of $400 \mu \mathrm{m}$ in diameter was analyzed. The XPS spectra were calibrated to the energy of $\mathrm{C} 1 \mathrm{~s}$ of the $\mathrm{C}-\mathrm{C}$ bond $(284.8 \mathrm{eV})$. Concerning the Kratos Axis ultra spectrometer, the analyzed area of the samples was $300 \times 700 \mu \mathrm{m}^{2}$. Peaks were recorded with constant pass energy of $20 \mathrm{eV}$. For the $\mathrm{Ag} 3 \mathrm{~d}_{5 / 2}$ line the full width at half-maximum (FWHM) was $0.58 \mathrm{eV}$ under the recording conditions. The pressure in the analysis chamber was around $5 \times 10^{-9} \mathrm{mbar}$. Short acquisition time spectra were recorded before and after each normal experiment to check that the samples did not suffer from degradation during the measurements. The binding energy scale was calibrated using the $\mathrm{C} 1 \mathrm{~s}$ peak at $285.0 \mathrm{eV}$ from the hydrocarbon contamination invariably present. Core peaks were analyzed using a nonlinear Shirley-type background ${ }^{41}$. The 
peak positions and areas were optimized by a weighted least-squares fitting method using $70 \%$ Gaussian, 30\% Lorentzian line shapes. Quantification was performed on the basis of Scofield's relative sensitivity factors ${ }^{42}$. The curves fit for core peaks were obtained using a minimum number of components in order to fit the experimental curves.

\section{Electron Microscopy}

The chemical homogeneity and composition of the broken buttons were checked using an Energy Dispersive Analysis of X-Ray (EDX) Hitachi S4800 mounted on a Hitachi S2600N scanning electron microscope as well as aliquot of the powder after ball grinding. Powders, dispersed on carbon film, were analyzed on a Jeol 1200 EXII TEM at $100 \mathrm{kV}$, equipped with a Quemesa camera (SIS Olympus) with 11 Mpixel CCD detector.

\section{Conflicts of interest}

There are no conflicts to declare.

\section{Acknowledgements}

This work was supported by the CheMISyst labex ANR-10-LABX-05-01 and University of Montpellier and the authors would also like to thank Bertrand Rebière and Didier Cot (IEM, France) for SEM-EDX characterization, Jean-Olivier Durand for his help with the DLS.

\section{Notes and references}

1. C. Forman, I. K. Muritala, R. Pardemann and B. Meyer, Renew. Sust. Energ. Rev., 2016, 57, 1568-1579.

2. D. M. Rowe, Thermoelectric Handbook - Macro to Nano, CRC Press, Taylor and Francis,, 2006.

3. D. M. Rowe, Thermoelectrics and its energy harvesting - Materials, preparation, and characterization in thermoelectrics, CRC Press, Taylor and Francis,, 2012.

4. S. LeBlanc, S. K. Yee, M. L. Scullin, C. Dames and K. E. Goodson, Renew. Sust. Energ. Rev., 2014, 32, 313-327. 
5. M. I. Fedorov and G. N. Isachenko, Jpn. J. Appl. Phys., 2015, 54.

6. P. Hermet, M. Khalil, R. Viennois, M. Beaudhuin, D. Bourgogne and D. Ravot, RSC Adv., 2015, 5, 19106-19116.

7. M. Khalil, PhD, University of Montpellier, 2015.

8. M. Khalil, M. Beaudhuin, B. Villeroy, D. Ravot and R. Viennois, J. Alloy. Compd., 2016, 662, $150-156$.

9. S. Karuppaiah, M. Beaudhuin and R. Viennois, J. Solid State Chemist., 2013, 199, 90-95.

10. R. Esmilaire, M. Beaudhuin, P. Hermet, N. Fréty, D. Ravot and R. Viennois, Mater. Lett., $2015, \mathbf{1 3 8}, 222-224$

11. S. Perumal, S. Gorsse, U. Ail, M. Prakasam, B. Chevalier and A. M. Umarji, J. Mater. Sci., $2013,48,6018-6024$.

12. G. H. Kim, L. Shao, K. Zhang and K. P. Pipe, Nat. Mater., 2013, 12, 719-723.

13. O. Bubnova, Z. U. Khan, A. Malti, S. Braun, M. Fahlman, M. Berggren and X. Crispin, Nat. Mater., 2011, 10, 429-433.

14. M. N. Gueye, A. Carella, N. Massonnet, E. Yvenou, S. Brenet, J. Faure-Vincent, S. Pouget, F. Rieutord, H. Okuno, A. Benayad, R. Demadrille and J.-P. Simonato, Chem. Mater., 2016, 28, 3462-3468.

15. T. Park, C. Park, B. Kim, H. Shin and E. Kim, Energ. Environ. Sci., 2013, 6.

16. Y. Du, S. Z. Shen, K. Cai and P. S. Casey, Prog. Polym. Sci., 2012, 37, 820-841.

17. N. Toshima, Synthetic Met., 2017, 225, 3-21.

18. K. C. See, J. P. Feser, C. E. Chen, A. Majumdar, J. J. Urban and R. A. Segalman, Nano Lett., 2010, 10, 4664-4667.

19. M. Jørgensen, K. Norrman and F. C. Krebs, Solar energy materials and solar cells, 2008, 92, 686-714.

20. N. Toshima, K. Oshima, H. Anno, T. Nishinaka, S. Ichikawa, A. Iwata and Y. Shiraishi, Advanced materials, 2015, 27, 2246-2251.

21. T. Fukumaru, T. Fujigaya and N. Nakashima, Macromolecules, 2012, 45, 4247-4253. 
22. T. Baumgartel, C. von Borczyskowski and H. Graaf, Beilstein J. Nanotech., 2013, 4, $218-$ 226.

23. S. Killian, PhD, Der Technischen Fakultät der Friedrich-Alexander-Universität ErlangenNürnberg, 2013.

24. J. Idígoras, M. Godfroy, D. Joly, A. Todinova, P. Maldivi, G. Oskam, R. Demadrille and J. A. Anta, $R S C A d v ., 2015, \mathbf{5}, 68929-68938$.

25. N. W. Polaske, H. C. Lin, A. Tang, M. Mayukh, L. E. Oquendo, J. T. Green, E. L. Ratcliff, N. R. Armstrong, S. S. Saavedra and D. V. McGrath, Langmuir, 2011, 27, 14900-14909.

26. R. Gheonea, C. Mak, E. C. Crasmareanu, V. Simulescu, N. Plesu and G. Ilia, J. Chem., 2017, 2017, 1-7.

27. R. Bhure, T. M. Abdel-Fattah, C. Bonner, F. Hall and A. Mahapatro, Appl. Surf. Sci., 2011, 257, 5605-5612.

28. C. Nicosia and J. Huskens, Mater. Horiz., 2014, 1, 32-45.

29. V. Spampinato, M. A. Parracino, R. La Spina, F. Rossi and G. Ceccone, Front. Chem., $2016,4,8$.

30. G. H. Woehrle, L. O. Brown and J. E. Hutchison, J. Am. Chem. Soc., 2004, 127, 2172-2183.

31. L. A. Porter Jr., D. Ji, S. L. Westcott, M. Graupe, R. S. Czernuszewicz, N. J. Halas and T. R. Lee, Langmuir, 1998, 14, 7378-7386.

32. D. C. Kennedy, G. Orts-Gil, C.-H. Lai, L. M. ller, A. Haase, A. Luch and P. H. Seeberger, J. Nanobiotechnol., 2014, 12:59, 1-8.

33. Y. Yu, C. E. Rowland, R. D. Schaller and B. A. Korgel, Langmuir, 2015, 31, 6886-6893.

34. J. M. Buriak, Chem. Rev., 2002, 102, 1271-1308.

35. M. Rosso-Vasic, E. Spruijt, B. van Lagen, L. De Cola and H. Zuilhof, Small, 2008, 4, 1835 1841.

36. J. Ma, Y. Gu, L. Shi, L. Chen, Z. Yang and Y. Qian, Journal of Alloys and Compounds, 2004, 376, 176-179.

37. J. Lua, H. Yang, B. Liu, J. Han and G. Zou, Materials chemistry and physics, 1999, 59, 101-106. 
38. S. Hallmann, M. J. Fink and B. S. Mitchell, J. Mater. Res., 2011, 26, 1052-1060.

39. C. M. Hessel, M. R. Rasch, J. L. Hueso, B. W. Goodfellow, V. A. Akhavan, P. Puvanakrishnan, J. W. Tunnel and B. A. Korgel, Small, 2010, 6, 2026-2034.

40. J. Rodriguez-Carvajal, presented in part at the Satellite meeting on powder diffraction of the XV congress of the IUCr, Toulouse, France, 1990.

41. D. A. Shirley, Physical Review B, 1972, 5, 4709.

42. J. H. Scofield, Journal of Electron Spectroscopy and Related Phenomena, 1976, 8, 129137. 